\title{
foF2 seismo-ionospheric effect analysis: actual data and numerical simulations
}

\author{
E. V. Liperovskaya ${ }^{1}$, P.-F. Biagi ${ }^{2}$, C.-V. Meister ${ }^{3}$, and M. V. Rodkin ${ }^{4}$ \\ ${ }^{1}$ Institute of Physics of the Earth of the Russian Academy of Sciences, Bolshaya Gruzinskaya 10, 123995 Moscow, Russia \\ ${ }^{2}$ Physics Department, University of Bari, 70126 Bari, Italy \\ ${ }^{3}$ Project Physics of Stellar and Planetary Atmospheres, An der Sternwarte 16, 14482 Potsdam, Germany \\ ${ }^{4}$ Geophysical Centre of the Russian Academy of Sciences, Molodejnaya 3, 117296 Moscow, Russia
}

Received: 14 July 2008 - Accepted: 20 August 2008 - Published: 11 December 2008

\begin{abstract}
On the background of seasonal and heliogeomagnetic disturbances, disturbances of the ionization density near noon connected to earthquakes are investigated. The study is performed for some tens of earthquakes with magnitudes $M>5$ and depths $h<70 \mathrm{~km}$, which occur at distances from the vertical sounding stations "Tokyo", "Akita", and "Yamagawa" not larger than $R=\exp (M)+150 \mathrm{~km}$. The analysis is performed using data registered by the three sounding stations every hour during 30 years. Especially methods of the statistical analysis are applied to search for regularities in the lithospheric-ionospheric links before and after earthquakes. The growth of the mean ionization density $N$ at the F-layer electron density maximum Fof2 five-three days before earthquakes, and the decrease of $N$ approaching the eruption and during a few days after the earthquake is investigated in dependence on both the magnitude of the earthquake $M$ and the ionization density $N$ near the F-layer maximum. It is found that some days before earthquakes the decrease of the ionization density $\Delta N$ is proportional to the values of $M$ and $N$. After the earthquakes, $\Delta N$ depends much weaker on the magnitude $M$, and it is not influenced by the ionization density $N$. The variations of the ionization density three days before earthquakes do not depend on $N$ and $M$ (within the same order of approximation).
\end{abstract}

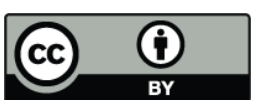

Correspondence to: C.-V. Meister (c.v.meister@skmail.ikp.physik.tudarmstadt.de)

\section{Introduction}

Almost all investigations performed up to now with respect to the lithospheric-atmospheric-ionospheric links had the aim to search for ionospheric precursors of earthquakes, and to solve the problem of the short-time prediction of earthquakes. Thus it was found, that the ionospheric precursors in different regions on the Earth have a very difficult temporal and spatial structure. Maximum effects are not always obtained above the epicentres, and the amplitude of the precursor does not always increase when the time approaches the eruption (Pulinets and Boyarchuk, 2004; Liu et al., 2006; Liperovsky et al., 2002; Singh et al., 2004). Thus, sometimes the conclusions of the investigations concerning regularities of the ionospheric precursors of earthquakes seem to be contradictory. In the present work, variations of the foF2-frequency, that means the ionization density at the F2-layer maximum, some days before and some days after earthquakes are analysed using data of the Japanese vertical sounding stations "Tokyo", "Akita", and "Yamagawa".

The main aim of the work is the search for regularities of lithospheric-ionospheric links connected to earthquakes using different vertical sounding stations. The interest is in the dependence of seismo-ionospheric effects on the magnitude of the earthquakes and on the ionization density at the F-layer maximum.

\section{Experimental data and method of analysis}

In the present work, ionospheric measurements of the foF2frequency obtained every hour by the stations "Akita" (latitude $=39.4^{\circ} \mathrm{N}$, longitude $\left.=140.1^{\circ} \mathrm{E}, 1957-1988\right)$ "Tokyo" ("Kokubunji", latitude $=35.7^{\circ} \mathrm{N}$, longitude $=139.5^{\circ} \mathrm{E}$, 1957-1990), and "Yamagawa" (latitude $=31.1^{\circ} \mathrm{N}$, longitude

Published by Copernicus Publications on behalf of the European Geosciences Union. 
$\left.=130.4^{\circ} \mathrm{E}, 1957-1988\right) \mathrm{http}: / /$ spidr.ngdc.noaa.gov/spidr/ are used.

Earthquakes with magnitudes $M>5$ and depths of the epicenters smaller than $70 \mathrm{~km}$ are considered. Searching for earthquake precursors, it is always of large importance to know, at which distances from the epicentres the effects are registered. Here, it is assumed that the studied phenomena of the F-layer are caused by acoustic and electromagnetic disturbances which are excited in the whole earthquake preparation region. The dimension of the earthquake preparation region is estimated by the Dobrovolsky formula $R_{D} \sim \exp (M)$ (Dobrovolsky et al., 1979). Disturbances caused by earthquakes may propagate up into the atmosphere and ionosphere not strongly vertically, but under a small tilding angle to the vertical. The maximum of the F-layer is situated at an altitude of the order of $300 \mathrm{~km}$. Further, it is suggested that the acoustic or electromagnetic disturbances have yet a sufficiently large amplitude, if the tangens of the angle between the propagation direction and the vertical is smaller than 0.5. Thus searching for earthquake precursors, events with distances between the epicenters and the vertical sounding stations less than $L=R_{D}+150 \mathrm{~km} \approx 500 \mathrm{~km}$ have to be taken into account. Therefore, concerning the stations "Tokyo" and "Akita", earthquakes situated at distances $R<R_{D}+150 \mathrm{~km}=500 \mathrm{~km}$ are analysed. The station "Yamagawa" is located in the south of the Isle of Kjushju, and thus the ocean coincides with large parts of the preparation regions of the appearing earthquakes. Thus, in this case earthquakes at $R \leq R_{D}$ are studied.

First rough earthquake investigations showed for the stations "Tokio", "Akita", and "Yamagawa", that no seismoionospheric effects are obtained for events with depths larger than $70 \mathrm{~km}$ and distances $R$ larger than considered here. In the present work, the dependence of the seismo-ionospheric effects on the hypocenter and the distance between the vertical sounding station and the epicenter is not studied. In cases when on one and the same day more than one earthquake occurred, all the earthquakes of the day are considered as one event.

The day-time ionospheric F-layer forms under the influence of the solar radiation with wavelengths of $14-80 \mathrm{~nm}$ on the neutral atmosphere. Thus, the atmospheric ionization is mainly connected with the ionization of oxygen $\mathrm{O}$ and nitrogen molecules $\mathrm{N}_{2}$. The altitude and the value of the main electron density maximum of the F-layer, which is characterized by the critical frequency foF2, depend on the competition between the ionization processes by the solar radiation and the recombination. The ionization intensity $N$ in the F-layer maximum satisfies the relation $N=\pi m(\mathrm{foF} 2)^{2} / e^{2}$, where $e$ is the elementary charge and $m$ the electron mass. For the study of the ionization density, in this work, first the hourly mean values were found, and then the average value for the hours between 11:00 LT and 16:00 LT is chosen. At this time, on the one side, the maximum plasma density of the layer is largest, and, on the other side, the area with max- imum ionization is situated at lower altitudes than at other hours. Thus one may conclude that seismo-ionospheric effects are largest during this time interval.

The ionization density of the F-layer possesses a rather difficult seasonal dependence. It has two maxima, in spring and in autumn, and two minima, in winter and in summer. Besides, it is influenced by the 11-years and the 27-days solar activity cycles. To reduce the influence of the season and the 27-days solar cycle, the average value of the ionization density $N_{15 \text { days }}(i)$ over the time interval of 15 days (starting with the day $i-7$ and ending with the day $i+7$ ) is subtracted from the mean value. The difference $\Delta N(i)=N(i)-N_{15}$ days $(i)$ depends yet on the 11 years solar activity cycle. To diminish also this effect, $\Delta N(i)$ is normalized by the mean square value of $\Delta N(i)$, that means by $\sigma(\Delta N(i))$. Thus, every day of the analysed time interval is characterized by the normalized function $\Delta N_{\text {norm }}(i)=\Delta N(i) / \sigma(\Delta N(i))$. The values of $\Delta N_{\text {norm }}(i)$ already weakly depend on the season, and the $27-$ days and 11-years solar variations. The distribution function $\Delta N_{\text {norm }}$ is an almost normal one.

The temporal behaviour of the ionization density $N$ shows sometimes very large positive and (more seldom) negative modifications, which are much larger than $\sigma(\Delta N(i))$. There were performed separate analyses to show if these modifications are indeed connected to earthquakes. During the here studied 12418 days, there are 120 days with large modifications. For these days the method of superposition of epoches is performed. For the day 0 the days with disturbances larger than $2 \sigma(\Delta N(i))$ are taken. Within the time intervals $(+5,-$ 5 ) of these days, there happened 28 earthquakes with $M>5$ - of a total of 358 events, 12 earthquakes with $M>5.5$ - of a total of 138, and 3 earthquakes with $M>6$ - of a total of 39 . Therefrom one may conclude that during the days of the strong modifications the number of earthquakes does not increase. Besides, the number of the days with strong modifications is rather small. So, in the present work searching for general regularities, these days are excluded from the analysis. It is estimated that the exclusion of the days does not change the final result of our statistics by more than $8 \%$.

The ionospheric effects of earthquakes have to be found also on the background of solar and geomagnetic disturbances. Thus only days with not too strong disturbances are taken into account. Of course, there do not exist generally accepted criteria which disturbances should be excluded from the analysis. In the present work days with Wolf numbers $W>200$ are neglected. As known, ionospheric disturbances may continue to exist for days after strong geomagnetic disturbances. Thus, days with $\Sigma K_{p}>25$, and the following day in each case, are not taken into account too. Here it has to be mentioned that a large part of the strong modifications, 83 out of 120 , were possibly caused by large solar activity with $W>200$ and increased geomagnetic distrubances of $\Sigma K_{p}>25$. For the analysis, the remaining series of the ionization density $N$, the differences to the 15-days mean values $\Delta N$, and the normalized values $\Delta N_{\text {norm }}$ are used. Of course, 


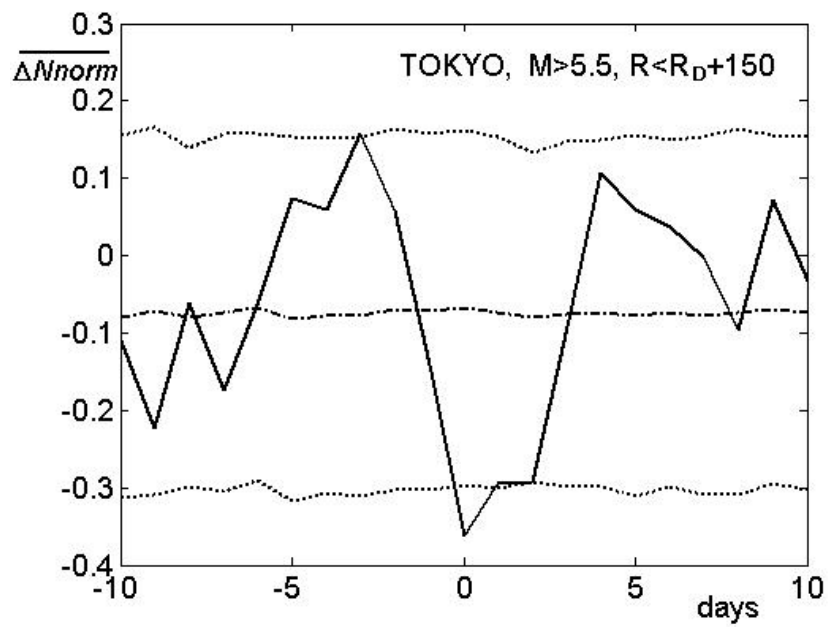

Fig. 1. Results of the superposition of epoches for $\Delta \overline{N_{\text {norm }}}$ found for 66 earthquakes of magnitude $M>5.5$ registered by the station "Tokyo". The day 0 is the day of the earthquakes.

there are series with lacks of datas. Consequently, from the initially 12418 data to be studied, there are finally analysed only half of them.

\section{Results of the superposition of epoches for different vertical sounding stations}

The method of the superposition of epoches is applied for the function of the normalized values $\Delta N_{\text {norm }}(i)$ of the differences between the ionization density and its 15-days mean values. The superposition of epoches is performed for the time intervals of 21 days, that means within the interval $(-10$, +10 ), for earthquakes with magnitudes $M>5.5$ registered by the stations "Tokyo", "Akita" and "Yamagawa". So the functions $\Delta \bar{N}_{\text {norm }}$ are found. For the station "Tokyo" there are considered 66 earthquakes, for "Akita" - 84 events, and for "Yamagawa" 21 events. The results of the analyses are presented in Figs. 1-3. It is to be seen, that, on the average, the value of $\Delta \bar{N}_{\text {norm }}$ grows on the days $(-5,-4,-3)$, and it decreases on the days $(0,+1,+2)$.

The sounding station "Tokyo" is situated at the region of intersection of three tectonic plates. This seems to be the reason for the fact that seismo-ionospheric phenomena found during the study of data of "Tokyo" are more strong than such phenomena found using data of the stations "Akita" and "Yamagawa". The here obtained seismo-ionospheric effects seem to depend on the Earth's core near the sounding stations. For data of "Tokyo" the increase and decrease of $\Delta \bar{N}_{\text {norm }}$ is even obtained for earthquakes with magnitudes $5.5>M>5.0$ (see Fig. 4). For the related superposition of epoches, 82 events existed. Performing the analysis for such rather weak earthquakes with $5.5>M>5.0$, time intervals

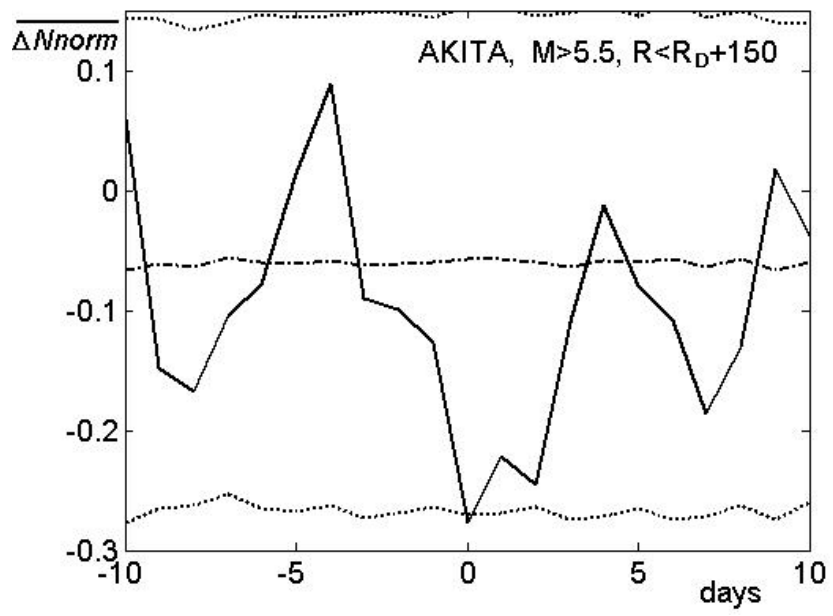

Fig. 2. Results of the superposition of epoches for $\Delta \overline{N_{\text {norm }}}$ found for 84 earthquakes of magnitude $M>5.5$ registered by the station "Akita". The day 0 is the day of the earthquakes. The dash-dotted line gives the mean values over the whole time interval of the 30 analysed years. The dotted line shows $95 \%$ of the reliability of the results of the analysis. The interval of reliability is found by Monte Carlo method.

$(-7,+7)$ around stronger earthquakes with $M>5.5$ are neglected.

The thin full lines in Figs. 1-5 give the mean value of $\Delta \bar{N}_{\text {norm }}$. In the case of "Tokyo", the decrease of $\Delta \bar{N}_{\text {norm }}$ after the earthquakes is also considerable. Applying the superposition of epoches method, data for every day are available, in the average, for 67 events.

The reliability interval is calculated modeling the background distribution with the help of a random process. It is assumed, that the earthquakes are homogeneously distributed within the observational time interval. Using a generator of random numbers, days of virtual earthquakes are chosen. The number of virtual earthquakes equals the number of real ones. Then, the superposition of epoches method is applied to the virtual earthquakes in the same way as to the real earthquakes. This procedure is repeated for a thousand of series of virtual earthquakes.

In Fig. 4, results of the superposition of epoches for earthquakes with $M>5.0$ near the station "Tokyo" are presented. For the analysis, 172 earthquakes are considered. From Fig. 2 follows that seismo-ionospheric effects occur most intensively on the days $(-3),(0)$ and $(+1)$. Let us study the dependence of the seismo-ionospheric effects on these days on the magnitude of the earthquakes and on the ionization density. For the superposition of epoches, the normalized differences $\Delta N_{\text {norm }}$ are used. Further, the non-normalized values $\Delta N$ are taken into account. 


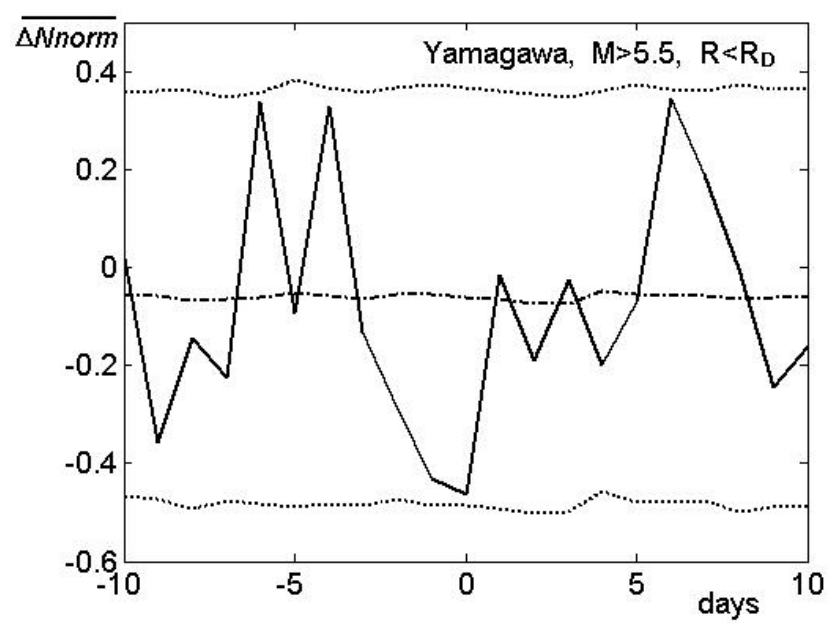

Fig. 3. Results of the superposition of epoches for $\Delta \overline{N_{\text {norm }}}$ found for 21 earthquakes of magnitude $M>5.5$ registered by the station "Yamagawa". The day 0 is the day of the earthquakes. The dashdotted line gives the mean values over the whole time interval of the 30 analysed years. The dotted line shows $95 \%$ of the reliability of the results of the analysis. The interval of reliability is found by Monte Carlo method.

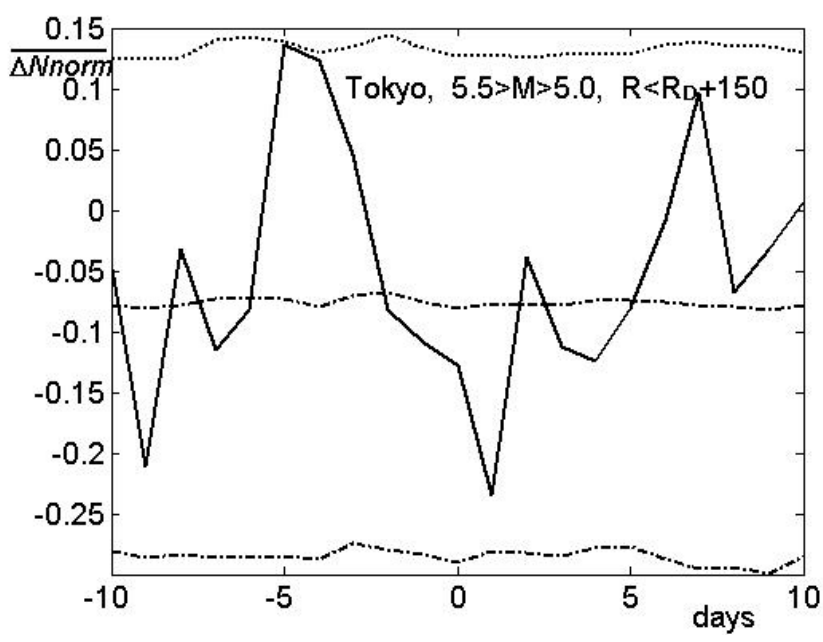

Fig. 4. Results of the superposition of epoches for $\Delta N_{\text {norm }}$ found for 82 earthquakes of magnitude $M>5.5$ registered by the station "Tokyo". Time intervals $(-7,+7)$ near earthquakes with $M>5.5$ are not taken into account. The day 0 is the day of the earthquakes. The dash-dotted line gives the mean values over the whole time interval of the 30 analysed years. The dotted line shows $95 \%$ of the reliability of the results of the analysis. The interval of reliability is found by Monte Carlo method.

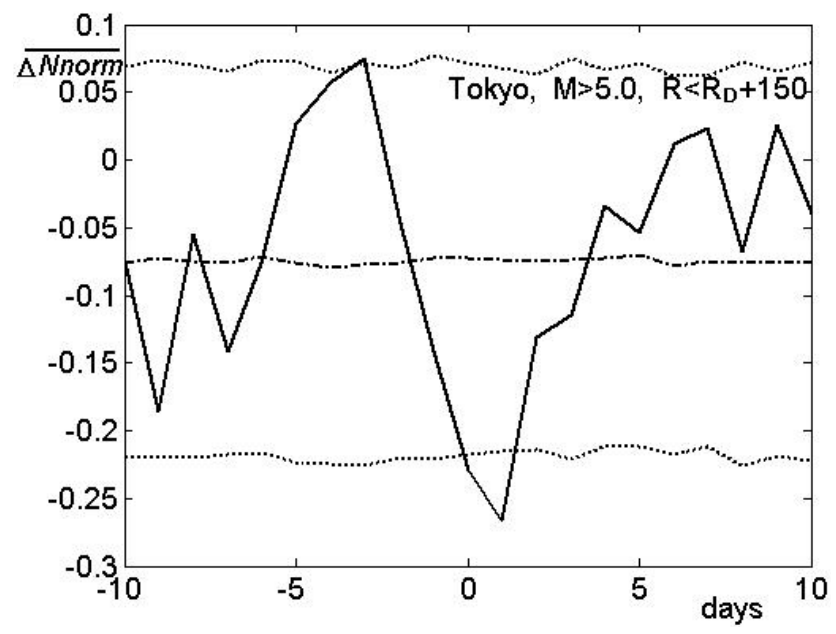

Fig. 5. Results of the superposition of epoches for $\Delta \overline{N_{\text {norm }}}$ found for 172 earthquakes of magnitude $M>5.0$ registered by the station "Tokyo". Time intervals $(-7,+7)$ near earthquakes with $M>5.5$ are not taken into account. The day 0 is the day of the earthquakes. The dash-dotted line gives the mean values over the whole time interval of the 30 analysed years. The dotted line shows $95 \%$ of the reliability of the results of the analysis. The interval of reliability is found by Monte Carlo method.

\section{Investigation of the dependence of the variations of the ionization density of the F-layer maximum on the ionization density and the magnitude of the earth- quake some hours before the eruption}

Now, for the analysis, earthquakes are taken into account, which happened in the time interval of $24 \mathrm{~h}$ after the daily measurements lasting from 11:00 UT till 16:00 LT, or lasting from 02:00 UT till 07:00 UT. First rough studies showed that, during time intervals of days, the mean value of the amplitude of the decrease of the ionization density does not depend on the time before the eruption.

Figure 6 shows the function $\Delta N(N)$ for the selected earthquakes. One may suggest, that seven variations with $|\Delta N|>5 \times 10^{5}$ are caused by non-seismic reasons, as they are obtained for all Japanese stations, for "Tokyo", "Akita", "Yamagawa", and "Vakkanai". These seven measurements are excluded from the analysis. Using the method of smallest squares, the regressive dependence $\Delta N(N)=-0.04 \times N+1.2 \times 10^{4}$ is obtained. The correlation coefficient between $\Delta N$ and $N$ equals $r=-0.22$. As 172 earthquakes are taken into account, the confidence of the dependence $\Delta N(N)$ is larger than $95 \%$.

Studying the dependence of the variation of ionization density $\Delta N$ on the magnitude of the earthquake $M$, one obtains $\Delta N(M)=-3.4 \times 10^{4} \times M+1.6 \times 10^{5}$ (see Fig. 7). The correlation coefficient between $\Delta N$ and $M$ equals $r=-0.10$, that means the reliability of the result is about $90 \%$. 


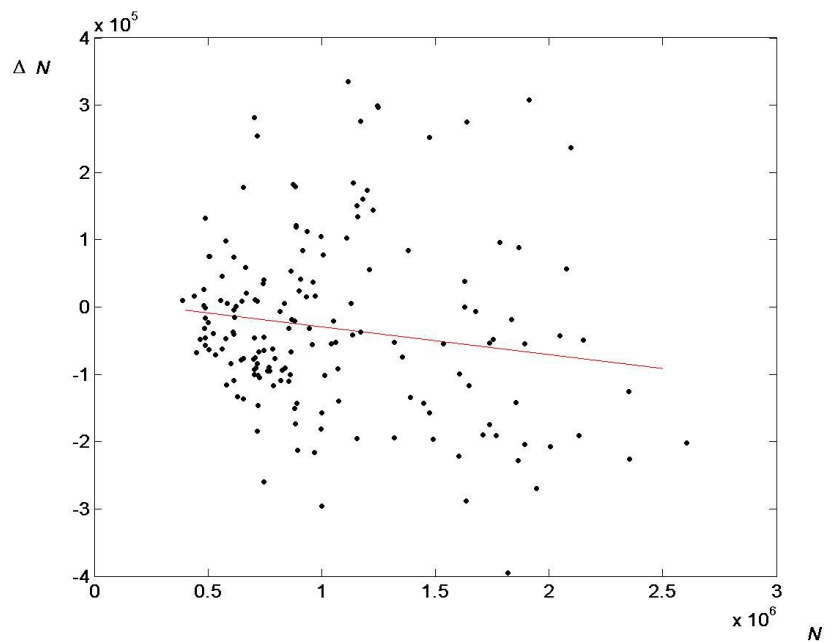

Fig. 6. Dependence of the variation of ionization density $\Delta N(N)$ on the ionization density $N$ in the F-layer maximum $24 \mathrm{~h}$ before the earthquakes. The red line shows the regressive dependence.

5 Investigation of the dependence of the variations of the ionization density of the F-layer maximum on the ionization density and the magnitude of the earthquake some hours after the eruption

Let us consider ionospheric phenomena occurring some hours after earthquakes, that means on the day $(+1)$. Performing first rough studies it was found, that during the first day after an earthquake, the mean value of the amplitude of the decrease of the ionization density does not depend on the time of the earthquake after the eruption. Figure 8 shows that the density indeed decreases on the day $(+1)$ with the time. Investigating the dependence $\Delta N(N)$, analogously to the discussion in the previous section, cases of very strong variations of the density - here with $|\Delta N|>4 \times 10^{5} \mathrm{~cm}^{-3}-$ are excluded from the analysis. As result of the analysis, one finds a correlation coefficient between $\Delta N$ and $N$ of $r=-$ 0.07. As 186 earthquakes are taken into account, the obtained effect is very weak. The mean decrease of the ionization density is about $-2.8 \times 10^{4} \mathrm{~cm}^{-3}$.

Further, the dependence of the variation of the ionization density $\Delta N$ on the magnitude of the earthquake $M$ is studied. Here variations $\Delta N>4 \times 10^{5} \mathrm{~cm}^{-3}$ are neglected. For the correlation coefficient between $\Delta N$ and $M$, a value of $r=-0.11$ is found. Consequently the confidence of the result is about $90 \%$. The dependence $\Delta N(N)$ satisfies the relation $\Delta N(N)=-2.0 \times 10^{3} \times M-2.0 \times 10^{4}$. Thus, the decrease of the ionization density after the earthquake depends much more weakly on the magnitude of the event than the decrease before the earthquake.

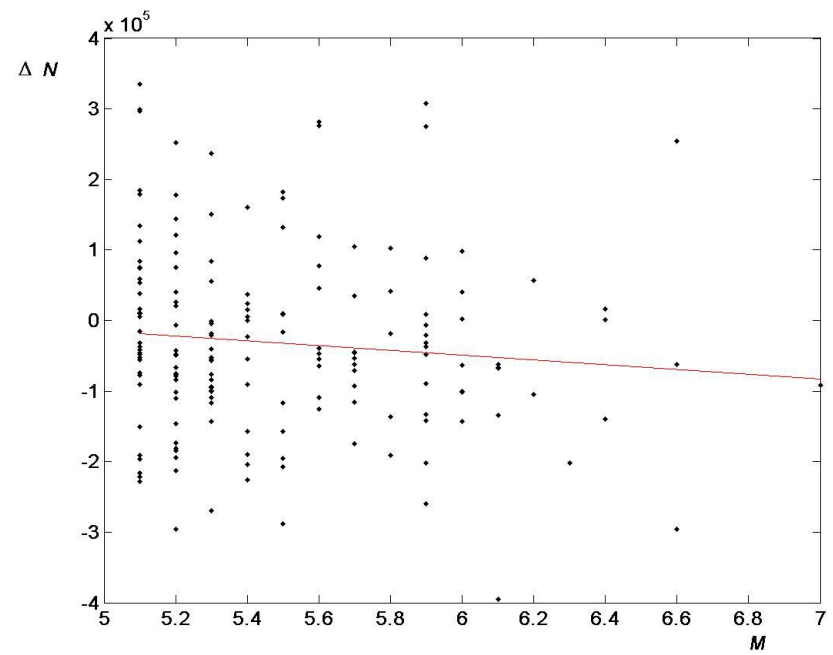

Fig. 7. Dependence of the variation of ionization density $\Delta N(M)$ on the magnitude $24 \mathrm{~h}$ before the earthquakes. The red line shows the regressive dependence.

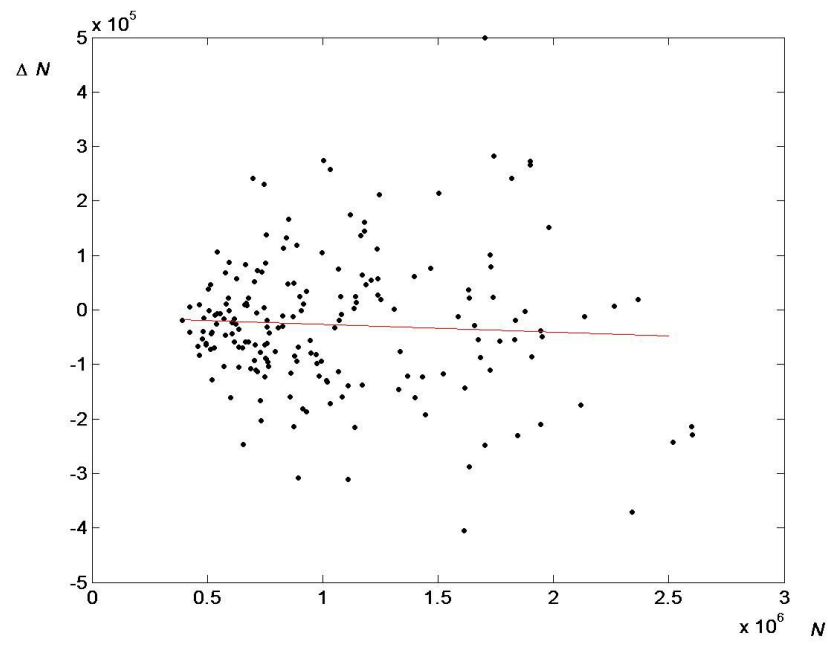

Fig. 8. Dependence of the variation of ionization density $\Delta N(N)$ on the ionization density $N$ in the F-layer maximum $24 \mathrm{~h}$ after the earthquakes. The red line shows the mean value of the decrease of $\Delta N$.

6 Investigation of the dependence of the variations of the ionization density of the F-layer maximum on the ionization density and the magnitude of the earthquake three days before the eruption

Finally we analogously consider ionospheric phenomena occurring during the first three days before earthquakes, that means on the days $(-3,-2,-1)$ (see Fig. 5). Here it is found that maximum increases of the ionization density are obtained on the day (-3). Investigating the dependence $\Delta N(N)$ 


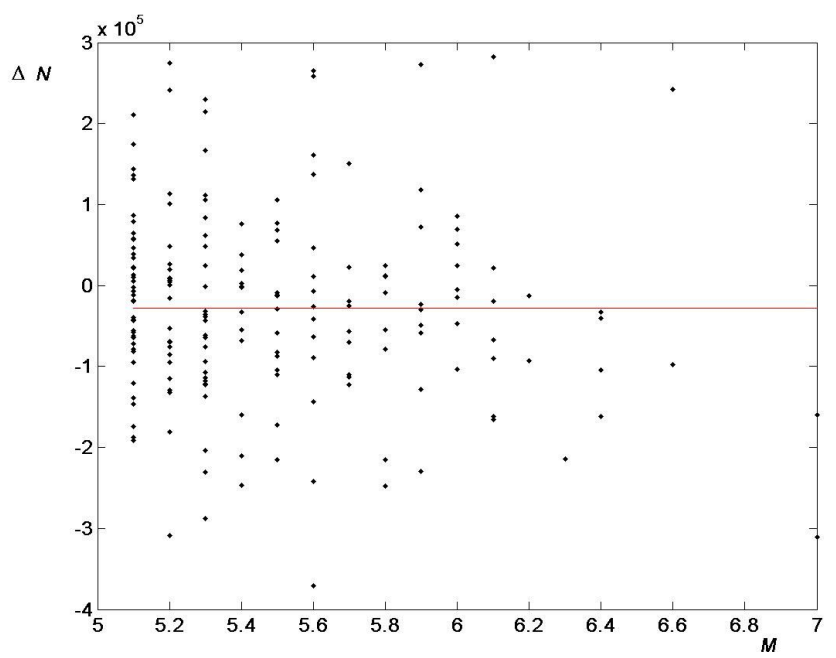

Fig. 9. Dependence of the variation of ionization density $\Delta N(M)$ on the magnitude $24 \mathrm{~h}$ after the earthquakes. The red line shows the mean decrease of $\Delta N$ after the earthquake.

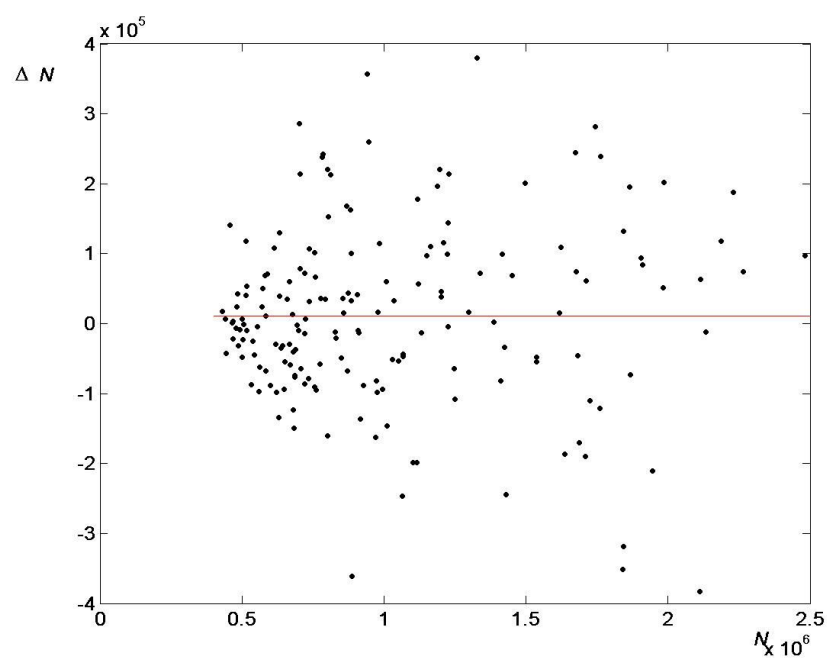

Fig. 10. Dependence of the variation of ionization density $\Delta N(N)$ on the ionization density $N$ in the F-layer maximum during the third days before the earthquakes. The red line shows the mean value of the increase of $\Delta N$ on the day (-3).

on the day (-3), two cases of very strong variations of the density with $|\Delta N|>4 \times 10^{5} \mathrm{~cm}^{-3}$ are excluded from the analysis. As result, one obtains a correlation coefficient $r$ of only -0.03 between $\Delta N$ and $N$. The mean increase of the ionization density is about $1.1 \times 10^{4} \mathrm{~cm}^{-3}$. In Fig. 10, the dependence $\Delta N(N)$ is presented.

Further, the dependence of the variation of the ionization density $\Delta N$ on the magnitude of the earthquake $M$ is studied. Here variations $\Delta N>4 \times 10^{5} \mathrm{~cm}^{-3}$ are again excluded from the consideration (that means in two cases). For the

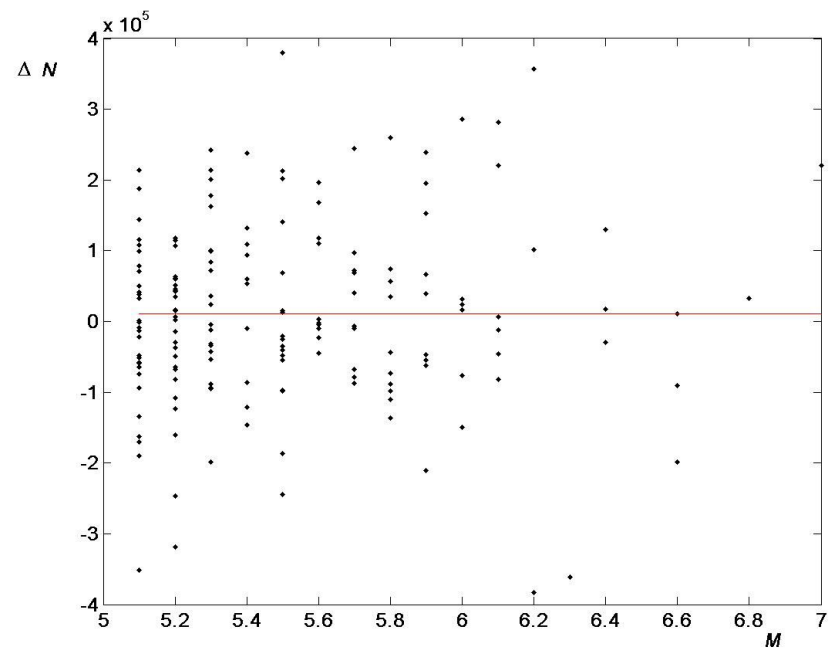

Fig. 11. Dependence of the variation of ionization density $\Delta N(M)$ on the magnitude three days before the earthquakes. The red line shows the mean increase of $\Delta N$ on the day (-3).

correlation coefficient between $\Delta N$ and $M$ a value $r=-0.06$ is found. As only 172 days are taken into account, the phenomenon seems not to be so important. From Fig. 11 follows that for earthquakes with a magnitude $M>6$ no increase of $\Delta N$ is found. And indeed, calculating the mean value of the increase for $6.0>M>5.0$, one obtains $1.1 . \times 10^{4} \mathrm{~cm}^{-3}$, which is about two times the mean value for all earthquakes considered.

\section{Discussion of the results and conclusions}

In the present work, the temporal behaviour of the ionization density in the F2-layer maximum is studied on the basis of measurements by the vertical sounding stations "Tokyo", "Akita" and "Yamagawa" during 30 years. As result, some regressive relations for the dependence of the variations of the ionization density on the ionization density in the Flayer maximum and on the growth rate of the earthquakes are found. Different regularities of the ionization density before and after earthquakes are described. As result follows that, in connection to earthquakes, different lithospheric-ionospheric links exist. A first coupling mechanism seems to be realized some days before earthquakes. Another coupling mechanism occurs especially during the fifth to third day before the eruptions. And a third mechanism takes place some hours after the earthquakes. The first and second mechanisms seem to be completely different, while the first and third mechanisms seem to be somewhat similar, or to have at least similar physical consequences.

The detailed analysis of the three physical mechanisms using data of the vertical sounding station "Tokyo" showed, that the ionization density $N$ in the ionospheric F-layer 
decreases some hours before the earthquakes. With a confidence of about $90 \%$, the dependence of the decrease on the magnitude of the earthquakes may be described by a linear function. And this decrease also depends almost linearly (with a realization probability of $95 \%$ ) on the ionization density of the ionosphere.

For the decrease of the ionization density a few days after earthquakes also a dependence on the magnitude of the earthquake was obtained. The confidence of the here proposed analytical formula seems to be of the order of $90 \%$. No direct dependence on the ionization density could be shown for the decrease of the ionization density after the earthquakes.

For the growth of the ionization density about three days before earthquakes, no reliable linear dependence on the magnitude or the ionization density could be found. There seems to exist a non-linear indirect dependence of the ionization density on the magnitude. The growth of the ionization density is obtained before eruptions with a magnitude $6>M>5$, and it is not found for magnitudes $M>6$. May be, in cases of earthquakes with $M>6$ three days before the eruption already the other physical mechanism is active, which causes a decrease of the density.

The authors of the present paper hope that the dependencies between the variations of the ionization density of the F-layer and the ionization density itself as well as between the variations and the magnitude of the earthquake obtained will be proven and refined in future analysing also data of other vertical sounding stations besides "Tokyo", "Akita", and "Yamagawa". This approach will help to verify proposed theoretical models of the physical processes of lithosphere-ionosphere coupling.

Edited by: M. Contadakis

Reviewed by: V. Bogdanov and another anonymous referee

\section{References}

Dobrovolsky, I. R., Zubkov, S. I., and Myachkin, V.I.: Estimation of the size of the earthquake preparation zones, Pageoph., 117, 1025-1044, 1979.

Liperovskaya, E. V., Bogdanov, V. V., Biagi, P.-F., Meister, C.V., Liperovsky, V. A., and Rodkin, M. V.: Day-time variations of foF2 connected to strong earthquakes, EGU 2008-A-02292, XY0382, 2008.

Liperovsky, V. A., Meister, C.-V., Popov, K. V., Liperovskaya, E. V., Molchanov, O. A., and Silina, A. S.: On the time scales of some seismo-ionospheric effects, Seismo-Electromagnetics: Lithosphere-atmosphere-ionosphere coupling, edited by: Hayakawa, M. and Molchanov, O. A., Tokyo, TERRAPUB, 325-327, 2002.

Liu, J. Y., Chen, Y. I., Chuo, Y. J., and Chen, C. S.: A statistical investigation of preearthquake ionospheric anomaly, J. Geophys. Res., 111, A05304, doi:10.1029/2005JA011333, 2006.

Pulinets, S. A. and Boyarchuk, K. A.: Ionospheric precursors of earthquakes, Springer, Berlin, Heidelberg, New York, 2004.

Singh, B., Kushwah, V., Singh, O. P., Lakshmi, D. R., and Reddy, B. M.: Ionospheric perturbations caused by some major earthquakes in India, Phys. Chem. Earth, 29, 537-550, 2004. 